\title{
The quadrupole moment of slowly rotating fluid balls
}

\author{
Michael Bradley*1 and Gyula Fodor ${ }^{\dagger 2}$ \\ ${ }^{1}$ Department of Physics, Umeå University, SE-901 87 Umeå, Sweden \\ ${ }^{2}$ KFKI Research Institute for Particle and Nuclear Physics, H-1525, Budapest 114, P.O.B. 49, Hungary
}

\begin{abstract}
In this paper we use the second order formalism of Hartle to study slowly and rigidly rotating stars with focus on the quadrupole moment of the object. The second order field equations for the interior fluid are solved numerically for different classes of possible equations of state and these solutions are then matched to a vacuum solution that includes the general asymptotically flat axisymmetric metric to second order, using the Darmois-Israel procedure. For these solutions we find that the quadrupole moment differs from that of the Kerr metric, as has also been found for some equations of state in other studies. Further we consider the post-Minkowskian limit analytically. In the paper we also illustrate how the relativistic multipole moments can be calculated from a complex gravitational potential.

PACS numbers: 04.40.Dg, 04.25.-g, 04.20.-q
\end{abstract}

\section{INTRODUCTION}

Sources for the Kerr metric have been sought for long, but with little success. The only known exact solution that can be matched to the Kerr metric seems to be the rotating dust disk, with maximal allowed angular momentum, found by Neugebauer and Meinel [1]. Some numerical studies with different equations of state for the source find quadrupole moments differing from that of the Kerr metric, see e.g. [2, [3, 4, , 5]. In some recent papers [6, 7] it is shown that in the rigidly rotating case incompressible fluids and fluids with polytropic equation of state cannot be sources of the Kerr metric in the postMinkowskian limit. These results are in accordance with the general expectation that the ellipsoidal shape of the rotating fluid ball produces an extra contribution to the quadrupole moment which should also be present in the corresponding quadrupole moment of the external field [4, 8]. For a general review of relativistic rotating stars see [9].

In the present work we use the second order formalism for slowly and rigidly rotating stars, developed by Hartle [10], to study the quadrupole moment and its deviation from that of the Kerr metric for different classes of possible equations of state. For some earlier applications of the formalism see, e.g., [2, 3, 11] and for a comparison with numerical solutions of the full Einstein equations see [4]. The relativistic multipole moments of the vacuum exterior metric up to order two are calculated using an algorithm developed in [12].

The field equations for the fluid region to second order in the small rotational parameter $\Omega$ will be solved numerically using fourth order Runge-Kutta. When imposing an equation of state this system can be rewritten as a first order system of ordinary differential equations for nine functions, but a subsystem for six of these func-

\footnotetext{
*electronic address: michael.bradley@physics.umu.se

†electronic address: gfodor@rmki.kfki.hu
}

tions will be sufficient to study for our purposes. Assuming regularity at the centre the solutions will depend on three constants of integration, corresponding to zeroth order central density or pressure, the magnitude of the angular velocity and one more second order small constant. If the solutions are required to be asymptotically flat this second order constant will be determined in terms of the other constants. Due to scaling invariance in the angular velocity it may be given a fixed value in the numerical runs. Hence, given an equation of state, we need only vary the central pressure or density when scanning the solution space. The solutions are then matched to a second order axisymmetric vacuum solution using the Darmois-Israel procedure [13, 14, 15]. This metric includes the general second order asymptotically flat stationary axisymmetric vacuum solution as a special case.

We also consider the post-Minkowskian limit analytically by expanding the field equations in the small parameter $\lambda \equiv G M / r_{1} c^{2}$ and make a comparison with the results of $[\underline{6}, 7]$.

The paper is organized as follows: In section [II the method is briefly described and the field equations are presented. Also the second order vacuum metric is given and its relativistic multipole moments up to order two are calculated. The matching procedure is described in section [II] and the integration constants for the vacuum solution are solved for in terms of the values of the interior solution on the matching surface. In section IV the equations are rewritten in a form suitable for numerical integration. The results of the numerical runs are given in section $\nabla$ and finally a post-Minkowskian analysis is made in section VI.

\section{PRELIMINARIES}

To second order the metric of a slowly rotating axisymmetric object, both in the interior fluid region and 
the outside vacuum region, can be written as

$$
\begin{aligned}
d s^{2}= & (1+2 h) A^{2} d t^{2}-(1+2 m) \frac{1}{B^{2}} d r^{2}- \\
& (1+2 k) r^{2}\left[d \theta^{2}+\sin ^{2} \theta(d \varphi-\omega d t)^{2}\right],
\end{aligned}
$$

where $\omega$ is first order and $h, m$ are $k$ are second order in the rotational parameter [10]. The requirements of regularity at the centre and asymptotic flatness imply that the first order function $\omega$ depends on $r$ only. The second order functions $h, m, k$ can be given as

$$
\begin{aligned}
h & =h_{0}+h_{2} P_{2}(\cos \theta) \\
m & =m_{0}+m_{2} P_{2}(\cos \theta) \\
k & =k_{2} P_{2}(\cos \theta)
\end{aligned}
$$

where $h_{0}, m_{0}$ and $h_{2}, m_{2}, k_{2}$ are functions of $r$ only and $P_{2}(x)=\frac{1}{2}\left(3 x^{2}-1\right)$ is the second order Legendre polynomial. This result follows from reflection symmetry in the equatorial plane, from that the equations for $h, m$ and $k$ separate with the ansätze $h=\sum_{i=0}^{\infty} h_{i}(r) P_{i}(\cos \theta)$ etc., and from the fact that there are no inhomogeneous terms containing $\omega$ in the equations for $h_{i}, k_{i}$ and $m_{i}$ for $i>2$. For more details see [10].

The matching of the two spacetime regions happens via the application of a coordinate transformation $\varphi \rightarrow$ $\varphi+\Omega t$ in the fluid region. It means that the inner fluid region rotates with respect to the distant stationary observers with angular velocity $\Omega$. This parameter $\Omega$ is considered to be the small expansion parameter with respect to which $\omega$ is first order and the other corrections $h, m, k$ are second order. In addition to this, we can also rescale the interior time coordinate first by a zeroth order constant $c_{4}$, and then later by a second order small constant $c_{3}$, i.e. $t \rightarrow c_{4}\left(1+c_{3}\right) t$.

The matter content of the interior is modelled by a perfect fluid

$$
T_{a b}=(\rho+p) u_{a} u_{b}-p g_{a b}
$$

The coordinate system used in (1) is assumed to be comoving with the fluid, i.e. the 4-velocity is assumed to possess the form

$$
u^{a}=\left(1 / \sqrt{g_{00}}, 0,0,0\right)=((1-h) / A, 0,0,0)
$$

which also implies that the shear of the fluid is zero so it rotates rigidly.

\section{A. The field equations}

In this subsection we list the field equations relevant to various orders. A similar system of equations with a slightly different choice of variables was given in [16]. If no equation of state is specified then the only equation one gets to zeroth order of the rotational parameter is the pressure isotropy condition $G^{1}{ }_{1}=G^{2}{ }_{2}$ which reads as

$$
B \frac{d^{2} A}{d r^{2}}+\frac{d(r A)}{d r} \frac{d(B / r)}{d r}+\frac{A}{r^{2} B}=0 .
$$

Making use of $G^{0}{ }_{0}=T_{0}^{0}$ and $G^{1}{ }_{1}=T^{1}{ }_{1}$ the energy density and pressure of the non-rotating configuration reads as

$$
\begin{aligned}
& \rho_{0}=\frac{1}{r^{2}}\left[1-\frac{d\left(r B^{2}\right)}{d r}\right], \\
& p_{0}=\frac{1}{r^{2}}\left[\frac{B^{2}}{A^{2}} \frac{d\left(r A^{2}\right)}{d r}-1\right] .
\end{aligned}
$$

To first order in the rotation parameter the only relation follows from $G_{0}^{3}=0$

$$
\frac{d}{d r}\left(r^{4} \frac{B}{A} \frac{d \omega}{d r}\right)+4 r^{3} \omega \frac{d}{d r}\left(\frac{B}{A}\right)=0 .
$$

The second order Einstein equations yield the following conditions. From $G_{2}^{1}=0$ one gets

$$
r \frac{d}{d r}\left(h_{2}+k_{2}\right)+r\left(h_{2}-m_{2}\right) \frac{1}{A} \frac{d A}{d r}-h_{2}-m_{2}=0 .
$$

The pressure isotropy condition in the angular directions, $G_{2}^{2}=G_{3}^{3}$, gives

$$
6\left(h_{2}+m_{2}\right)-r^{4} \frac{B^{2}}{A^{2}}\left(\frac{d \omega}{d r}\right)^{2}+4 r^{3} \omega^{2} \frac{B}{A} \frac{d}{d r}\left(\frac{B}{A}\right)=0 .
$$

The equality of the pressure in the angular and radial directions, i.e. $G^{1}{ }_{1}=G^{2}{ }_{2}$ gives two equations. After eliminating the derivative of $h_{2}$ using (8) one obtains from the $P_{2}(\cos \theta)$ part

$$
\begin{gathered}
2 r \frac{B^{2}}{A} \frac{d A}{d r}\left(r \frac{d k_{2}}{d r}-m_{2}\right)-2 r^{2} B h_{2} \frac{d}{d r}\left(\frac{B}{r}\right)+ \\
m_{2}-4 k_{2}-5 h_{2}-\frac{1}{3} r^{4} \frac{B^{2}}{A^{2}}\left(\frac{d \omega}{d r}\right)^{2}=0,
\end{gathered}
$$

while the $\theta$-independent part gives an equation for $m_{0}$ and $h_{0}$.

The energy density function can be decomposed as $\rho=$ $\rho_{0}+\rho_{2}$, where $\rho_{2}=\rho_{20}+\rho_{22} P_{2}(\cos \theta)$ and $\rho_{20}$ and $\rho_{22}$ are second order small functions of the coordinate $r$. The analogous decomposition of the pressure is similarly given by $p=p_{0}+p_{2}=p_{0}+p_{20}+p_{22} P_{2}(\cos \theta)$. We get one more equation for $m_{0}$ and $h_{0}$ if we assume that the equation of state $\rho=\rho(p)$ is unchanged to second order. For details see [11].

\section{B. Equations of state}

To complete the system one more equation, e.g. an equation of state

$$
\rho=\rho(p),
$$


has to be specified. In this paper we will consider equations of state in the form:

$$
\rho=d_{1} p+d_{2}\left(\frac{p}{p_{c}}\right)^{1 / \gamma}+d_{3}
$$

Here $p_{c}$ is the central pressure. Equation (12) includes some of the more used approximations for the equation of state of dense stars, like Newtonian polytropes, relativistic polytropes with $d_{1}=1 /(\gamma-1)$, linear ones as well as the incompressible case.

We use units such that the speed of light $c=1$ and $8 \pi G=1$. If then the unit of length is taken as $\alpha$ meter, the relation between our units and SI units are given by

$$
\begin{aligned}
\rho_{S I} & =5.358 \times 10^{25} \frac{\rho}{\alpha^{2}} \mathrm{~kg} / \mathrm{m}^{3} \\
x_{S I} & =4.813 \times 10^{42} \frac{x}{\alpha^{2}} \mathrm{kgm}^{-1} \mathrm{~s}^{-2} \\
d_{1 S I} & =d_{1}
\end{aligned}
$$

where $x=\rho c^{2}, p, d_{2}$ or $d_{3}$.

Note that $m_{0}$ and $h_{0}$ do not appear in equations (5), (7), (8), (9), (10) and (11) and hence this subsystem for $A, B, \omega, m_{2}, k_{2}$ and $h_{2}$ decouples. Notice also that the equations can be solved order by order and that the equations contain $m_{2}$ only algebraically. In section $\nabla$ the system will be reformulated as a coupled system of six first order ordinary differential equations. Due to the requirement of a regular centre the solutions to this subsystem will only depend on three constants of integration.

\section{Vacuum metric}

In the exterior vacuum region we will use a frame adapted to the asymptotically non-rotating observer. Solving the field equations detailed in Section IIA by imposing $p=\rho=0$ the metric functions for the vacuum region are given as follows $[10,17]^{1}$

$$
\begin{gathered}
A^{2}=B^{2}=1-2 M / r \\
\omega=\frac{2 a M}{r^{3}},
\end{gathered}
$$

\footnotetext{
1 To make the norm of the timelike Killing field equal to unity at spatial infinity, the expression of $h_{0}$ in [17] has been slightly modified through a second order coordinate transformation of the time coordinate.
}

$$
\begin{aligned}
h_{0}= & -m_{0}=\frac{1}{r-2 M}\left(\frac{a^{2} M^{2}}{r^{3}}+c_{2}\right) \\
h_{2}= & 3 c_{1} r(2 M-r) \log \left(1-\frac{2 M}{r}\right)+a^{2} \frac{M}{r^{4}}(M+r) \\
& +2 c_{1} \frac{M}{r}\left(3 r^{2}-6 M r-2 M^{2}\right) \frac{r-M}{2 M-r} \\
& +\left(1-\frac{2 M}{r}\right) r^{2} q_{1} \\
k_{2}= & 3 c_{1}\left(r^{2}-2 M^{2}\right) \log \left(1-\frac{2 M}{r}\right)-a^{2} \frac{M}{r^{4}}(2 M+r) \\
& -2 c_{1} \frac{M}{r}\left(2 M^{2}-3 M r-3 r^{2}\right)+\left(2 M^{2}-r^{2}\right) q_{1} \\
m_{2}= & 6 a^{2} \frac{M^{2}}{r^{4}}-h_{2} .
\end{aligned}
$$

In this approximation, the slowly rotating solution is characterized by the mass $M$, the first order small rotation parameter $a$, and the second order small constants $c_{1}, c_{2}$ and $q_{1}$. When $q_{1}$ takes the value zero the metric is known to be the general asymptotically flat stationary and axisymmetric vacuum metric to second order (see e.g. [18]). It can be easily checked that the solution is of Petrov type $\mathrm{D}$ only if both $c_{1}$ and $q_{1}$ are zero. The metric is then equivalent to the Kerr metric to second order with mass $M \rightarrow M-c_{2}$.

When $q_{1} \neq 0$ the metric cannot be asymptotically flat. It is important to keep in mind, however, that without the inclusion of this constant the matching conditions on the zero pressure surface are overdetermined in general [17, 19].

\section{Multipole moments of the vacuum metric}

The notion of relativistic gravitational multipole moments for static asymptotically flat vacuum spacetimes was developed in 20 by Geroch and later extended to the stationary case in 21] and 22] by Hansen and Thorne. These moments are defined on the 3 -space of the timelike Killing trajectories.

A 3-space $(\mathcal{M}, h)$ with positive definite metric $h$ is said to be asymptotically flat if it can be conformally mapped to a manifold $(\tilde{\mathcal{M}}, \tilde{h})$ with the following properties

(i) $\tilde{\mathcal{M}}=\mathcal{M} \cup \Lambda$, where $\Lambda$ is a single point,

(ii) $\left.\tilde{\Omega}\right|_{\Lambda}=\left.\tilde{\Omega}_{, i}\right|_{\Lambda}=0,\left.\tilde{D}_{i} \tilde{D}_{j} \tilde{\Omega}\right|_{\Lambda}=\left.\tilde{h}_{i j}\right|_{\Lambda}$ where $\tilde{h}_{i j}=\tilde{\Omega}^{2} h_{i j}$.

From the timelike Killing vector field $K^{a}$ one constructs the two scalar functions $f=K^{a} K_{a}$ and $\psi$, where the later is obtained from the curl of $K^{a}, \psi_{, a}=$ $\epsilon_{a b c d} K^{b} K^{c ; d}$, that is a gradient due to the vacuum equations. The complex gravitational potential then reads

$$
\xi=\frac{1-\mathcal{E}}{1+\mathcal{E}}
$$


in terms of the Ernst potential $\mathcal{E}_{\tilde{\xi}}=f+i \psi$. It is given the conformal weight $-1 / 2$, so that $\tilde{\xi}=\tilde{\Omega}^{-1 / 2} \xi$. For axisymmetric spacetimes the metric is completely determined by the value of the potential on the axis of symmetry [23].

The multipole tensors on the 3 -space of timelike Killing trajectories, with coordinates $x^{i}, i=1,2,3$ and metric given by the projection operator $h_{a b}=-f g_{a b}+K_{a} K_{b}$, are then defined recursively as

$$
\begin{aligned}
& P^{(0)}\left(x^{i}\right)=\xi, \quad P_{j}^{(1)}\left(x^{i}\right)=\xi_{, j}, \\
& P_{k_{1} k_{2} \ldots k_{n+1}}^{(n+1)}\left(x^{i}\right)=D_{<k_{n+1}} P_{k_{1} \ldots k_{n}>}^{(n)}- \\
& \frac{1}{2} n(2 n-1) R_{<k_{1} k_{2}} P_{k_{3} \ldots k_{n+1}>}^{(n-1)}
\end{aligned}
$$

where $<k_{1} \ldots k_{n+1}>$ denotes the symmetric and tracefree part. $D_{i}$ and $R_{i j}$ are the covariant derivative and Ricci tensor, respectively, with respect to the 3 -metric $h_{i j}$ [21]. The $\tilde{P}_{k_{1} \ldots k_{n}}^{(n)}$ are defined correspondingly in terms of the tilded quantities.

For axisymmetric spacetimes the multipole moments are given entirely in terms of the scalar moments defined as

$$
P_{n}=\left.\frac{1}{n !} \tilde{P}_{k_{1} \ldots k_{n}}^{(n)} n^{k_{1}} \ldots n^{k_{n}}\right|_{\Lambda}
$$

in terms of the axis vector $n^{i}$ [21].

In, e.g., [12] and 24] algorithms for calculating the multipole moments of a stationary axisymmetric spacetime are developed. We here use the method of [12] to calculate the moments up to second order. First the metric is transformed to the canonical form

$$
d s^{2}=f(d t-\tilde{\omega} d \varphi)^{2}-f^{-1}\left[e^{2 \gamma}\left(d \rho^{2}+d z^{2}\right)+\rho^{2} d \varphi^{2}\right],
$$

where $f, \gamma$ and $\tilde{\omega}$ are functions of $\rho$ and $z$, through the coordinate transformation

$$
\begin{aligned}
\rho= & \operatorname{Ar} \sin \theta\left[1+h_{0}+h_{2}+k_{2}-\frac{3}{2} \sin ^{2} \theta\left(h_{2}+k_{2}\right)\right] \\
z= & {\left[(r-M)\left(1+2 h_{0}-\frac{1}{2} \sin ^{2} \theta\left(h_{2}+2 k_{2}-m_{2}\right)\right)\right.} \\
& \left.+r^{2} A^{2}\left(h_{0, r}-\frac{1}{2} \sin ^{2} \theta\left(h_{2, r}+k_{2, r}\right)\right)\right] \cos \theta
\end{aligned}
$$

that holds to second order.

From the timelike Killing vector $K^{a}=\delta_{0}^{a}$ we find the two potentials

$$
f=K^{a} K_{a}=g_{00}=(1+2 h) A^{2}-r^{2} \omega^{2} \sin ^{2} \theta
$$

and

$$
\psi=-\frac{2 a M}{r^{2}} \cos \theta
$$

obtained from the curl of $K^{a}$.

Introduce the coordinates $\bar{\rho}=\rho /\left(\rho^{2}+z^{2}\right), \bar{z}=z /\left(\rho^{2}+\right.$ $\left.z^{2}\right)$. A suitable conformal factor is then $\tilde{\Omega}=\bar{r}^{2} \equiv \bar{\rho}^{2}+\bar{z}^{2}$ and hence $\tilde{\xi}=(1 / \bar{r}) \xi$. As shown in [12], the first (up to $n=3$ ) scalar moments $P_{n}$ are given by the coefficients $m_{n}$ in the expansion

$$
\tilde{\xi}(\bar{\rho}=0)=\Sigma_{n=0}^{\infty} m_{n} \bar{z}^{n}
$$

of $\tilde{\xi}$ on the axis, that in the original coordinates corresponds to $\theta=0$ ( or $\pi$ ). Expansion of $\xi$ on the axis in the original coordinates gives to second order in the rotational parameter

$$
\begin{aligned}
\xi= & \frac{M}{r-M}+i \frac{M a}{(r-M)^{2}}-\frac{M^{2} a^{2}}{r(r-M)^{3}}- \\
& \left(h_{0}+h_{2}\right) A^{2}\left(\frac{r}{r-M}\right)^{2} .
\end{aligned}
$$

From (19) one finds that $r=z+M$ to zeroth order, which is sufficient for the three last terms in (23) since they are already first and second order. The first term need to be expanded to second order in the rotational parameter, giving

$$
\begin{aligned}
\frac{M}{r-M}= & \frac{M}{z-2 h_{0}(r-M)-r^{2} A^{2} h_{0, r}}= \\
& \frac{M}{z}+\frac{M}{z^{2}}\left[2 h_{0}(r-M)+r^{2} A^{2} h_{0, r}\right]
\end{aligned}
$$

where once again $r=z+M$ can be used in all second order functions. Finally, expressing $\tilde{\xi}$ in $\bar{z}$, one obtains to second order

$$
\tilde{\xi}=M-c_{2}+i M a \bar{z}-M\left(a^{2}+\frac{16}{5} M^{4} c_{1}\right) \bar{z}^{2} .
$$

Hence the mass is given by $M-c_{2}$, the angular momentum by $J=M a$ and the quadrupole moment by $Q=-M\left(a^{2}+\frac{16}{5} M^{4} c_{1}\right)$. We will be interested in the relative deviation of the quadrupole moment from that of the Kerr metric

$$
\frac{\Delta Q}{Q} \equiv \frac{Q-Q_{K e r r}}{Q_{K e r r}}=\frac{16 M^{4} c_{1}}{5 a^{2}} .
$$

An expansion of the exterior metric for large $r$ gives the following leading terms of $g_{00}\left(\right.$ with $\left.q_{1}=0\right)$

$$
g_{00}=1-\frac{2 M\left(1-\frac{c_{2}}{M}\right)}{r}+\frac{2 M P_{2}(\cos \theta)\left(a^{2}+\frac{16}{5} M^{4} c_{1}\right)}{r^{3}},
$$

i.e., the associated Newtonian quadrupole moment reads as (cf., e.g. 25])

$$
Q_{11}=Q_{22}=-Q_{33} / 2=2 M\left(a^{2}+\frac{16}{5} M^{4} c_{1}\right)
$$

in an asymptotically Cartesian system with the 3 -axis along the axis of rotation. Hence it is, up to a factor of 4 , the same as the relativistic moment. 


\section{MATCHING}

We here briefly describe the matching procedure, in which the Darmois-Israel junction conditions [13, 14] are used. For more details the reader is referred to [11] and also to [15] for a general discussion on the matching of axisymmetric bodies in second order perturbation theory. In the fluid region, the matching surface $\mathcal{S}$ is defined by the condition of vanishing pressure, $p=0$. In the limit of no rotation, the matching surface is the 3 -dimensional cylinder $r=r_{1}$. For slow rotation the equation of the matching surface $\mathcal{S}$ is

$$
r=r_{1}+\xi
$$

with

$$
\xi=-\left[\frac{p_{20}+p_{22} P_{2}(\cos \vartheta)}{\frac{d p_{0}}{d r}}\right]_{\mid r=r_{1}} \equiv \xi_{0}+\xi_{2} P_{2}(\cos \vartheta)
$$

where the constants $\xi_{0}$ and $\xi_{2}$ are given by

$$
\begin{aligned}
\xi_{0}= & \frac{1}{12 r B \frac{d A}{d r} \frac{d}{d r}\left(\frac{A}{B}\right)} \times \\
& {\left.\left[12 r A^{2} \frac{d h_{0}}{d r}-12 m_{0} \frac{d}{d r}\left(r A^{2}\right)+r^{4}\left(\frac{d \omega}{d r}\right)^{2}\right]\right|_{r=r_{1}} }
\end{aligned}
$$

and

$$
\xi_{2}=-\left.\frac{\left(3 A^{2} h_{2}+r^{2} \omega^{2}\right)}{3 A \frac{d A}{d r}}\right|_{r=r_{1}}
$$

In the vacuum exterior region suitable hypersurfaces for matching are determined by

$$
r=r_{1}+\chi \equiv \chi_{0}+\chi_{2} P_{2}(\cos \vartheta),
$$

where $\chi_{0}$ and $\chi_{2}$ are constants to be determined by the matching conditions [26].

In order to find isometric embeddings of the matching surface $\mathcal{S}$ in the vacuum and fluid domains, we equate with each other the respective induced metrics $d s_{(v)}^{2}, d s^{2}$ and induced extrinsic curvatures $K^{(v)}, K$

$$
\left.d s_{(v)}^{2}\right|_{\mathcal{S}}=\left.\left.d s^{2}\right|_{\mathcal{S}} \quad K^{(v)}\right|_{\mathcal{S}}=\left.K\right|_{\mathcal{S}}
$$

where $K$ is defined by

$$
K \equiv K_{a b} d x^{a} d x^{b} \equiv h_{a}^{c} h_{b}{ }^{d} n_{(c ; d)} d x^{a} d x^{b}
$$

in terms of the unit normal $n_{a}$ of the matching surface $\mathcal{S}$ and the projection operator $h_{a}^{b}=n_{a} n^{b}+\delta_{a}^{b}$. To adjust the different coordinate systems with each other we apply a rigid rotation in the fluid region by setting $\varphi \rightarrow \varphi+\Omega t$ where $\Omega$ is a constant. Then we re-scale the interior time coordinate $t \in R$ by $t \rightarrow c_{4}\left(1+c_{3}\right) t$ with further zeroth and second order constants $c_{4}$ and $c_{3}$ to be determined from the matching conditions.
From the zeroth order matching conditions we get the following relations (all functions here and in the following first and second order relations are evaluated at the zeroth order matching surface $r=r_{1}$ ):

$$
\begin{gathered}
M=\frac{1}{2} r_{1}\left(1-B^{2}\right), c_{4}=\frac{B}{A}, \\
r_{1}=\frac{A}{2 B^{2} \frac{d A}{d r}}\left(1-B^{2}\right) .
\end{gathered}
$$

To first order we solve for $a$ and $\Omega$

$$
a=\frac{B r_{1}^{3}}{3 A\left(B^{2}-1\right)} \frac{d \omega}{d r}, \Omega=\frac{r_{1}}{3} \frac{d \omega}{d r}+\omega .
$$

From the second order equations we can solve for $c_{1}, c_{2}$, $c_{3}, q_{1}, \chi_{0}$ and $\chi_{2}$ as

$$
\begin{gathered}
c_{1}=\frac{B^{2}}{9 r_{1}^{2} A^{2}\left(B^{2}-1\right)^{6}}\left[r_{1}^{4} B^{2}\left(B^{4}-3\right)\left(\frac{d \omega}{d r}\right)^{2}\right. \\
\left.+36 A^{2} h_{2}\left(1-B^{4}\right)+72 A^{2} B^{2}\left(h_{2}+k_{2}\right)\right] \\
c_{2}=\frac{\xi_{0}}{2}\left(B^{2}-1+2 r_{1} B \frac{d B}{d r}\right)-r_{1} B^{2} m_{0}-\frac{r_{1}^{5}}{36} \frac{B^{2}}{A^{2}}\left(\frac{d \omega}{d r}\right)^{2} \\
q_{1}=\frac{r_{1}^{4}}{9 r_{1}^{2} A^{2}\left(B^{2}-1\right)^{6}}\left(\frac{d \omega}{d r}\right)^{2}+\frac{c_{2}}{r_{1} B^{2}}-h_{0} \\
+216 A^{2} B^{2} \ln B\left[2 B ^ { 2 } \left(h_{2}+k_{2}\left(B^{4}-1\right)\left(B^{4}-8 B^{2}+1\right)\right.\right. \\
36 h_{2} A^{2} B^{2}\left(B^{2}-1\right)\left(B^{4}+B^{2}-8\right)+ \\
r_{1}^{4}\left(\frac{d \omega}{d r}\right)^{2} B^{2}\left[\left(B^{2}-1\right)\left(2+11 B^{2}-7 B^{4}\right)+\right. \\
\left.\left.6 B^{2}\left(B^{4}-3\right) \ln B\right]\right\} \\
\chi_{0}=\xi_{0} \quad \text { and } \chi_{2}=\xi_{2},
\end{gathered}
$$

where $\xi_{0}$ and $\xi_{2}$ are obtained from (31) and (31). Note that we did not impose the equation of state (11) when calculating the matching conditions. Hence an appropriate matching can be done, i.e. the vacuum metric in section ПC is general enough for describing the exterior of any axisymmetric rigidly rotating perfect fluid ball up to second order.

\section{EQUATIONS AND BOUNDARY VALUES}

In this section we provide a reformulation of the field equations to a form more suitable for numerical integration. By doing this we can get higher precision at the origin where apparent singularities arise, moreover the freely specifiable constants are identified more easily this way. 


\section{A. Integrating the zeroth order field equation}

In order to simplify (5) it is convenient to redefine the functions $A, h, m$ and $k$ in terms of the function $\nu$ as

$$
A=e^{\nu}, \quad h=\tilde{h} e^{-2 \nu}, \quad m=\tilde{m} e^{-2 \nu}, \quad k=\tilde{k} e^{-2 \nu} .
$$

The equations simplify considerably due to the fact that only the derivative of $\nu$ will appear. Hence we introduce the function $z$ by

$$
\frac{z}{B}=r \frac{d \nu}{d r}+1
$$

Then the zeroth order equation (5) becomes first order in $z$ and algebraic in $B$ [27]

$$
B r \frac{d z}{d r}+2 B^{2}+z^{2}-4 B z+1=0
$$

furthermore, the pressure of the non-rotating configuration (6) takes the form

$$
p_{0}=\frac{1}{r^{2}}\left(2 B z-B^{2}-1\right) .
$$

\section{B. Series expansion around a regular centre}

For sufficiently regular configurations close to the centre the metric coefficients can be given as power series in $r$. Assuming that the central pressure and density are finite it follows that $B(0)=z(0)=1$. The assumption of smoothness of the configurations at the symmetry centre, in the spacetime sense, implies that the odd coefficients in the expansions of the basic variables are zero.

Hence, assuming a smooth centre in the spacetime sense, the odd powers will be omitted hereafter. Plugging the expressions

$$
\begin{aligned}
B & =1+b_{1} r^{2}+b_{2} r^{4}+\ldots \\
z & =1+z_{1} r^{2}+z_{2} r^{4}+\ldots \\
\omega & =\omega_{0}+\omega_{1} r^{2}+\omega_{2} r^{4}+\ldots \\
\tilde{h}_{2} & =h_{2}^{(0)}+h_{2}^{(1)} r^{2}+h_{2}^{(2)} r^{4} \ldots \\
\tilde{m}_{2} & =m_{2}^{(0)}+m_{2}^{(1)} r^{2}+m_{2}^{(2)} r^{4} \ldots \\
\tilde{k}_{2} & =k_{2}^{(0)}+k_{2}^{(1)} r^{2}+k_{2}^{(2)} r^{4} \ldots
\end{aligned}
$$

into the field equations together with the equation of state (11) justifies then that all coefficients can be given in terms of $z_{1}, \omega_{0}$ and $h_{1} \equiv h_{2}^{(1)}$.

To zeroth order one obtains

$$
h_{2}^{(0)}=m_{2}^{(0)}=k_{2}^{(0)}=0,
$$

then to second order

$$
\begin{aligned}
m_{2}^{(1)} & =k_{2}^{(1)}=-h_{2}^{(1)} \equiv-h_{1} \\
\omega_{1} & =\frac{2}{5} \omega_{0}\left(z_{1}-3 b_{1}\right), \quad b_{1}=-\frac{1}{6} \rho_{\mid 2 z_{1}}
\end{aligned}
$$

and finally to fourth order

$$
\begin{aligned}
b_{2}= & -\frac{b_{1}^{2}}{2}+\left.\frac{1}{10}\left(z_{1}^{2}+3 b_{1}^{2}-4 b_{1} z_{1}\right) \frac{d \rho}{d p}\right|_{2 z_{1}}, \\
z_{2}= & b_{1} z_{1}-\frac{z_{1}^{2}}{2}-b_{1}^{2} \\
h_{2}^{(2)}= & \frac{\left(3 z_{1}^{2}-16 z_{1} b_{1}+8 b_{1}^{2}-10 b_{2}\right)\left(\omega_{0}^{2}+3 h_{1}\right)}{42\left(z_{1}-b_{1}\right)} \\
& +\frac{\omega_{0}^{2}}{21}\left(17 b_{1}-5 z_{1}\right) \\
k_{2}^{(2)}= & \frac{\omega_{0}^{2}}{6}\left(z_{1}-3 b_{1}\right)+\frac{h_{1}}{2}\left(b_{1}-z_{1}\right)-h_{2}^{(2)}, \\
m_{2}^{(2)}= & \frac{2 \omega_{0}^{2}}{3}\left(z_{1}-3 b_{1}\right)-h_{2}^{(2)} \\
\omega_{2}= & \frac{\omega_{0}}{70}\left(z_{1}^{2}-36 z_{1} b_{1}+74 b_{1}^{2}-50 b_{2}\right) .
\end{aligned}
$$

The central density and pressure are given by

$$
\rho_{0 c}=-6 b_{1}, \quad p_{0 c}=2 z_{1} .
$$

This shows that for realistic configurations $b_{1}<0$ and $z_{1}>0$, consequently the $z_{1}-b_{1}$ term in the denominator of $h_{2}^{(2)}$ is nonvanishing.

\section{System of differential equations}

Motivated by the results of the previous section it is advantageous to define the new dependent variables $\beta, \zeta$, $\tilde{\omega}, \hat{\omega}, \hat{h}, \hat{k}$ and $\hat{m}$ through

$$
\begin{aligned}
B & =1+r \beta, \quad z=1+r \zeta, \\
\omega & =\omega_{0}+r \tilde{\omega}, \quad \omega_{, r}=\tilde{\omega}+\hat{\omega}, \\
\tilde{h}_{2} & =r \hat{h}, \quad \tilde{k}_{2}=r\left(r^{2} \hat{k}-\hat{h}\right), \quad \tilde{m}_{2}=r\left(r^{2} \hat{m}-\hat{h}\right) .
\end{aligned}
$$

The closed subsystem of equations (5), (7), (8), (9), (10) and (11) then gives six first order differential equations for the quantities $\beta, \zeta, \tilde{\omega}, \hat{\omega}, \hat{h}$ and $\hat{k}$, while $\hat{m}$ can be solved for algebraically. The equations for the zeroth order quantities are given by

$$
\begin{aligned}
& \frac{d \zeta}{d r}=-\frac{1}{r B}\left(-\zeta-3 r \zeta \beta+r \zeta^{2}+2 r \beta^{2}\right) \\
& \frac{d \beta}{d r}=-\frac{1}{2 r B}\left(r \rho_{0}+4 \beta+3 r \beta^{2}\right)
\end{aligned}
$$

where the density $\rho_{0}$ is given by equation of state $\rho_{0}=$ $\rho\left(p_{0}\right)$ with the pressure given by

$$
p_{0}=\frac{1}{r}\left(2 \zeta+2 r \zeta \beta-r \beta^{2}\right) .
$$

The equations for the first order quantities $\tilde{\omega}$ and $\hat{\omega}$ are

$$
\begin{aligned}
\frac{d \tilde{\omega}}{d r}= & \frac{\hat{\omega}}{r} \\
\frac{d \hat{\omega}}{d r}= & -\frac{1}{r B}\left[r\left(4 \omega_{0}+5 r \tilde{\omega}+r \hat{\omega}\right) \frac{d \beta}{d r}+4(2 \beta-\zeta) \omega_{0}\right. \\
& +(14 r \beta+4-5 r \zeta) \tilde{\omega}+(7 r \beta+5-r \zeta) \hat{\omega}]
\end{aligned}
$$


whereas the ones for the second order quantities $\hat{h}$ and $\hat{k}$ are long and hence are not given here. The corresponding homogeneous equations, given by putting $\omega=0$ in (8), (9) and (10), are

$$
\begin{aligned}
\frac{d \hat{h}_{\mathrm{h}}}{d r} & =\frac{2 r \hat{k}_{\mathrm{h}}-\beta \hat{h}_{\mathrm{h}}}{r(\beta-\zeta) B}+\frac{\hat{h}_{\mathrm{h}}}{\beta-\zeta} \frac{d \beta}{d r} \\
\frac{d \hat{k}_{\mathrm{h}}}{d r} & =\frac{r \hat{k}_{\mathrm{h}}(2 r \zeta-5 r \beta-3)+2 \hat{h}_{\mathrm{h}}(\beta-\zeta)}{r^{2} B} .
\end{aligned}
$$

These two last equations will be used when adjusting the parameters so that the solutions become asymptotically flat.

Boundary conditions at $r=0$ are given as

$$
\begin{aligned}
& \frac{d \beta}{d r}=b_{1}, \frac{d \zeta}{d r}=z_{1}, \frac{d \tilde{\omega}}{d r}=\frac{d \hat{\omega}}{d r}=\frac{2}{5} \omega_{0}\left(z_{1}-3 b_{1}\right), \\
& \frac{d \hat{h}}{d r}=h_{1}, \frac{d \hat{k}}{d r}=\frac{\omega_{0}^{2}}{6}\left(z_{1}-3 b_{1}\right)+\frac{h_{1}}{2}\left(b_{1}-z_{1}\right) .
\end{aligned}
$$

The constants $b_{1}=-\rho_{0 c} / 6$ and $z_{1}=p_{0 c} / 2$ are related through the equation of state (11), implying that we have three independent constants of integration (apart from possible constants in the equation of state). One of this $\left(h_{1}\right)$ will be determined through the matching conditions on the zero pressure surface in terms of the others when the requirement of asymptotic flatness is imposed.

There is a rescaling associated to the rescaling of the rotational parameter $\omega_{0}$, following the rule $\omega_{0} \rightarrow \gamma \omega_{0}$, which induces the transformation

$$
\begin{aligned}
& \omega, \tilde{\omega}, \hat{\omega} \rightarrow \gamma \omega, \gamma \tilde{\omega}, \gamma \hat{\omega} \quad \hat{h}, \hat{m}, \hat{k} \rightarrow \gamma^{2} \hat{h}, \gamma^{2} \hat{m}, \gamma^{2} \hat{k}, \\
& \beta, \zeta, r \rightarrow \beta, \zeta, r .
\end{aligned}
$$

Due to this scale invariance of the equations the constant $\omega_{0}$ can be fixed. All other configurations with a given equation of state and specified central pressure can be obtained by rescaling.

The rescaling of the radial coordinate $r \rightarrow \alpha r$ induces a change of the parameters in the equation of state, (12), given by

$$
d_{1} \rightarrow d_{1}, \quad d_{2}, d_{3}, p_{0 c} \rightarrow \frac{d_{2}}{\alpha^{2}}, \frac{d_{3}}{\alpha^{2}}, \frac{p_{0 c}}{\alpha^{2}} .
$$

The dependent variables then scale as

$$
\begin{aligned}
& \beta, \zeta, \tilde{\omega}, \hat{\omega}, \hat{m}, \hat{k} \rightarrow \frac{\beta}{\alpha}, \frac{\zeta}{\alpha}, \frac{\tilde{\omega}}{\alpha}, \frac{\hat{\omega}}{\alpha}, \frac{\hat{m}}{\alpha}, \frac{\hat{k}}{\alpha}, \\
& \omega, \omega_{0}, \hat{h} \rightarrow \omega, \omega_{0}, \alpha \hat{h} .
\end{aligned}
$$

Using this scale invariance we can set one of the constants in the equation of state to a fixed value.

\section{Asymptotically flat solutions}

Asymptotically flat solutions can be obtained by considering suitable linear combinations of homogeneous and particular solutions for $h_{2}$ and $k_{2}$, or equivalently for $\hat{h}$ and $\hat{k}$. These are solutions of the linear equations (58) and (59) and their corresponding inhomogeneous equations respectively. Since we solve the equations order by order, and hence $\hat{k}$ and $\hat{h}$ do not appear in the lower order equations, we can add any homogeneous solution to a given particular solution, i.e., $\hat{k}=\hat{k}_{p}+C \hat{k}_{h}$, $\hat{h}=\hat{h}_{p}+C \hat{h}_{h}$, where $C$ is an arbitrary constant and the subscripts $p$ and $h$ refer to particular and homogeneous solutions respectively. A solution to the field equations is asymptotically flat iff $q_{1}=0$. Now the expression (41) is also linear in $k_{2}$ and $h_{2}$, or equivalently in $\hat{k}$ and $\hat{h}$, it has a structure like

$$
q_{1}=\alpha_{1}+\alpha_{2} \hat{k}+\alpha_{3} \hat{h} .
$$

Hence

$$
\begin{aligned}
q_{1}= & \alpha_{1}+\alpha_{2}\left(\hat{k}_{p}+C \hat{k}_{h}\right)+\alpha_{3}\left(\hat{h}_{p}+C \hat{h}_{h}\right)= \\
& \alpha_{1}+\alpha_{2} \hat{k}_{p}+\alpha_{3} \hat{h}_{p}+C\left(\alpha_{2} \hat{k}_{h}+\alpha_{3} \hat{h}_{h}\right) \equiv \\
& q_{1 p}+C q_{1 h}
\end{aligned}
$$

and $q_{1}=0$ can be obtained by choosing $C=-q_{1 p} / q_{1 h}$. Also the constant $c_{1}($ (38), that is used to calculate the quadrupole moment, has a similar structure, and hence the resulting $c_{1}$ is given by $c_{1}=c_{1 p}+C c_{1 h}$.

\section{NUMERICAL SOLUTIONS}

In this section we consider solutions with equations of state (12), with special attention to the quadrupole moments and their deviation from that of the Kerr metric. In particular we determine the quantity $\Delta Q / Q$ defined in (26). The system was solved using fourth order Runge-Kutta. In scanning the parameter space, due to the scaling invariance, without loss of generality, we fixed $\omega_{0}=0.1$ and the method for obtaining asymptotically flat solutions, described in section IVD below, indirectly fixes the value of $h_{1}$. Hence, for a given equation of state, we only need to vary the central pressure, $2 z_{1}$. When considering different equations of state we use the scale invariance (62) and (63) to fix one of the constants $d_{2}$ or $d_{3}$. When performing the corresponding rescaling $r \rightarrow \alpha r$ the quantity $\Delta Q / Q$ is invariant.

The integrations were carried out until the zero pressure surface was reached. The accuracy of the code was checked for the Wahlquist solution [28].

\section{A. Incompressible fluids (interior Schwarzschild)}

This case was already considered in [3]. The numerically calculated quantity $\Delta Q / Q$, defined in (26), as a function of the central pressure is depicted in Figure 1 . In Figure 2 the same quantity is given as function of the radius of the corresponding non-rotating configuration. When the central pressure approaches infinity and the 


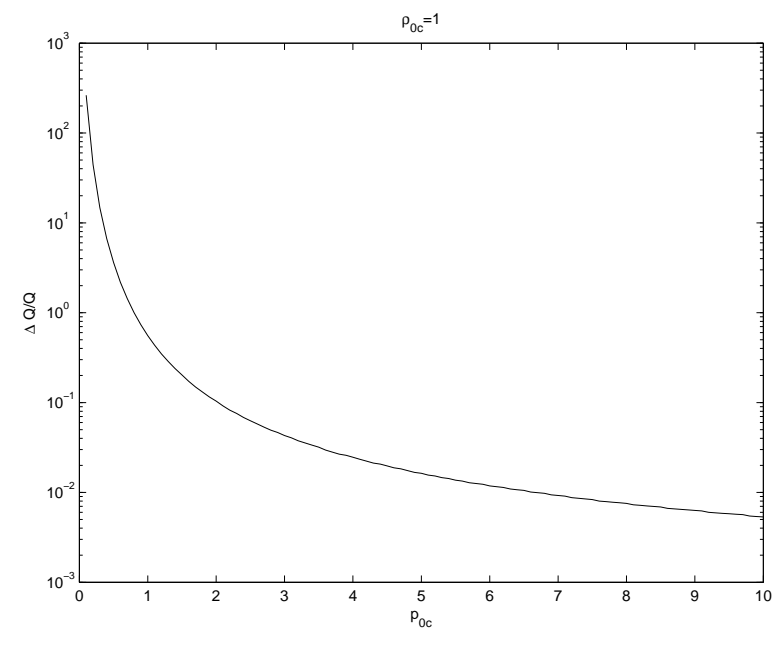

FIG. 1: The quantity $\Delta Q / Q$ as function of central pressure for a sequence of of fluid balls with constant density $\rho=1$.

radius the Buchdahl limit $\frac{9 M}{4}$, then $\Delta Q / Q$ approaches 0.0212. Due to the scaling invariance, (62) and (63), this limiting value is independent of the density.

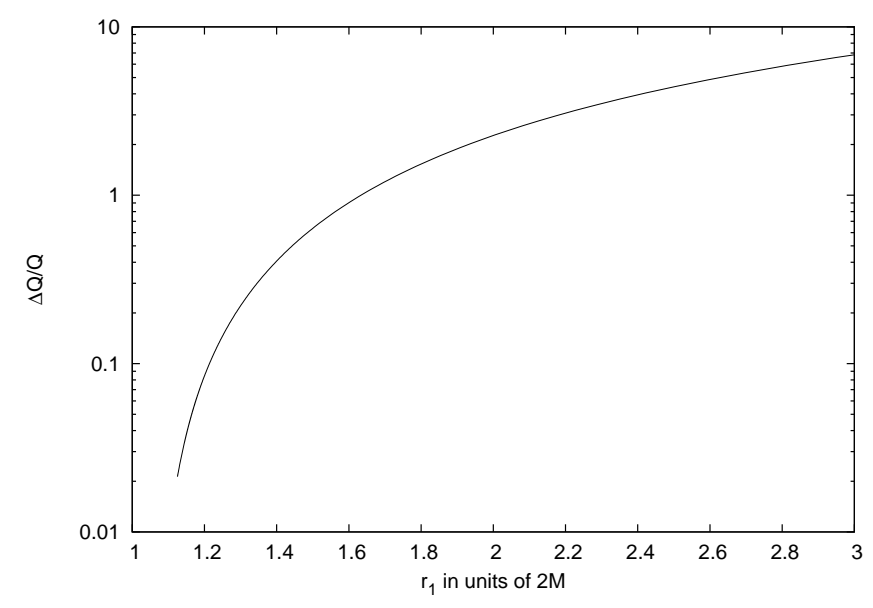

FIG. 2: The quantity $\Delta Q / Q$ as function of radius $r_{1} / 2 M$ for a sequence of of fluid balls with constant density $\rho=1$.

\section{B. Linear equations of state}

Linear equations of state

$$
\rho=d_{1} p+d_{3}
$$

are of interest not only because they could approximate some compact objects, but also since in this class one might have a chance of finding exact solutions, at least to zeroth order.

An exact spherically symmetric perfect fluid solution is given by the Whittaker metric [29], with equation of state $\rho=-3 p+\mu_{0}$. Its metric is

$$
d s^{2}=f_{0} d t^{2}-\frac{d r^{2}}{f_{0}\left(1-\kappa^{2} \mu_{0} r^{2} / 2\right)}-r^{2}\left(d \theta^{2}+\sin ^{2} \theta d \varphi^{2}\right)
$$

where

$$
f_{0}=1+\frac{1}{\kappa^{2}}\left[1-\arcsin \left(\kappa \sqrt{\frac{\mu_{0}}{2}} r\right) \sqrt{\frac{2}{\kappa^{2} \mu_{0} r^{2}}-1}\right]
$$

and the pressure is

$$
p=\frac{1}{2} \mu_{0}\left(1-\kappa^{2} f_{0}\right) .
$$

It is the non-rotating limit of the Wahlquist metric [28], that cannot be matched to an asymptotically flat vacuum region [17, 30]. However, there is another rotating generalisation of Whittaker that can be matched, although its closed-form is not known 31.

In the limit when the central pressure goes towards $\mu_{0} / 2$, corresponding to $\kappa \rightarrow 0$, the Whittaker metric approaches the anti-de Sitter spacetime and the radius $r_{1}$ blows up to infinity as $\sqrt{2 / \mu_{0}} / \kappa$, but $r_{1} / 2 M$ goes as $1+4 \kappa^{2} / \pi^{2}$, i.e. the surface approaches the event horizon. This is not in conflict with the Buchdahl limit [32], since this spacetime does not meet the usual physical requirements. Even if the these are weaker than in Buchdahl's original work, see e.g. [33], the central density becomes negative in the anti-de Sitter limit. For the corresponding second order rotating and asymptotically flat configuration we find that the quadrupole moment approaches that of the Kerr metric in this limit.

Similar results hold for all configurations with $d_{1}<-1$. For all these $\rho_{0 c}=-p_{0 c}$ when $p_{0 c}=d_{3} /\left(-d_{1}-1\right)$, and since the anti-de Sitter spacetime is a solution to our zeroth order equations and the solution is uniquely given by central pressure and density this is the resulting spacetime. Since the zero-pressure surface is further and further pushed outward when approaching the limit $p_{0 c}=d_{3} /\left(-d_{1}-1\right)$, all these configurations also become infinite in extension. The ratio $r_{1} / 2 M$ once again tends towards one, and we also reobtain that the quadrupole moment approaches that for Kerr. Even if these spacetimes are quite unphysical, a study of them still is helpful in understanding which conditions are needed for a successful matching to the Kerr metric.

When performing the numerical runs, due to the scaling invariance, (62) and (63), we put $d_{3}=1$. Negative values of $d_{3}$ are excluded if the surface density should be larger than or equal to zero, and configurations with $d_{3}=0$ are not finite in size [34]. The quantity $\Delta Q / Q$ for a sequence of values for $d_{1}$ are given in Figure 3 , As seen, for more realistic configurations with $d_{1} \geq 1$ it differs significantly from zero. 


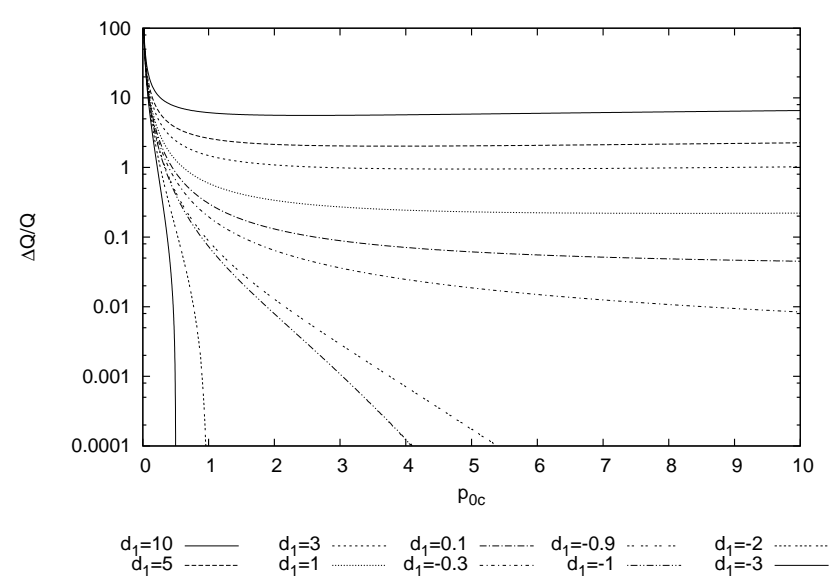

FIG. 3: The quantity $\Delta Q / Q$ as a function of central pressure for a sequence of fluid balls with linear equation of state $\rho=$ $d_{1} p+1$.

\section{Polytropes}

\section{Newtonian polytropes}

In a recent paper [7] it was shown that slowly and rigidly rotating polytropes cannot be sources of the Kerr metric in the post-Minkowskian limit. Here we numerically find that slowly and rigidly rotating polytropes with arbitrary strength of the gravitational field cannot be matched to Kerr either.

We use the equation of state $\rho=d_{2}\left(\frac{p}{p_{c}}\right)^{1 / \gamma}$ obtained by putting $d_{1}=d_{3}=0$ in (12). Due to the scaling invariance, (62) and (63), we put $d_{2}=1$. In Figure $4 Q / Q$ is shown as a function of the central pressure for a sequence of values of $\gamma$, including the physically interesting cases $\gamma=4 / 3$ and $\gamma=5 / 3$. As shown in [34] the configurations are finite in size for $\gamma>1.2295(1 / \gamma<0.7695)$ and infinite for $\gamma<1.2(1 / \gamma>0.833)$. In the interval in between the central pressure determines whether the configuration is finite or not. As seen $\Delta Q / Q$ never seems to approach zero. Furthermore, for physically reasonable configurations with $p_{c} \leq \rho_{c}=1$, the quantity differs significantly from zero.

\section{Relativistic polytropes}

For a fluid with one type of constituent particles, a relativistic polytrope is given by $p=C n^{\gamma}$, where $C$ is a constant, in terms of the particle density $n$ and the polytropic index $\gamma$. This equation is suitable, e.g., to describe an ideal degenerate neutron gas. Using the energy conservation equation for a perfect fluid it is then easy to show that the equation of state becomes

$$
\rho=\frac{1}{\gamma-1} p+d_{2} p^{1 / \gamma}
$$

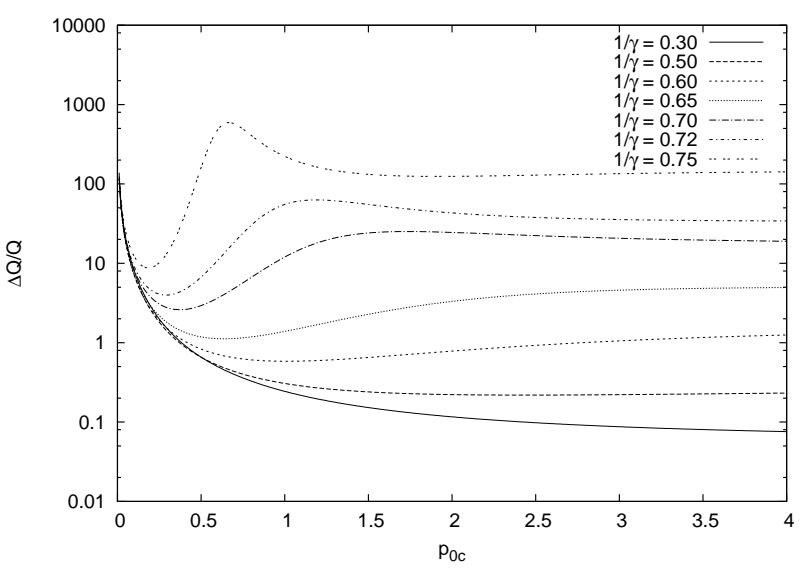

FIG. 4: The quantity $\Delta Q / Q$ as a function of central pressure for a sequence of Newtonian polytopes with equation of state $\rho=\left(\frac{p}{p_{c}}\right)^{1 / \gamma}$.

in terms of the pressure $p$. As seen the equation of state approaches a linear equation of state for large pressures and a Newtonian polytrope for low pressures. For a discussion of relativistic polytropes see. e.g., [35]. The results of the numerical runs are given in Figure 5 As expected the results are similar to those of the Newtonian polytropes.

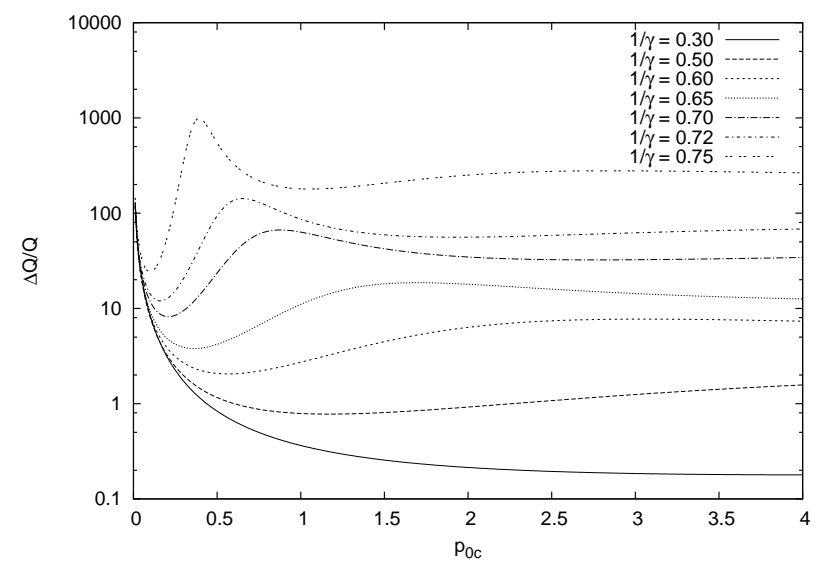

FIG. 5: The quantity $\Delta Q / Q$ as a function of central pressure for a sequence of relativistic polytropes with equation of state $\rho=\frac{1}{\gamma-1} p+\left(\frac{p}{p_{c}}\right)^{1 / \gamma}$.

\section{Combined linear and polytropic}

When adding a constant to the Newtonian polytropes in Figure 4 typically the local minima and maxima get less pronounced or disappear and for large central pressures the quadrupole moments get closer to that of the 
incompressible case, but never smaller. For combined linear and polytropic equations of state the curves become more similar to those of the relativistic polytropes in Figure 5. and when there is additative constant the limiting value of the quadrupole moment for large pressures once again gets closer to that of the incompressible case.

\section{THE POST-MINKOWSKIAN LIMIT}

Since we cannot solve the equations in the fluid region analytically, we look at the weak gravity limit by making an expansion in the small parameter $\lambda=M / r_{1}$, or in SI units $G M / r_{1} c^{2}$. Here $M$ is the mass of the fluid ball and $r_{1}$ is its radius. A similar study, using global harmonic coordinates and the Lichnerowicz matching conditions [36], was performed in [6] and [7].

From an expansion of the equations in this parameter it turns out that the pressure is one order higher in $\lambda$ than the density. This can also be understood in a Newtonian context where the virial theorem applied to a spherically symmetric configuration gives $\langle p\rangle=k \lambda\langle\rho\rangle$, where $k$ is a constant of order unity and $\langle p\rangle$ and $\langle\rho\rangle$ are the average values of pressure and energy density. By considering the balance between gravitational and centrifugal forces on the equator of a rotating Newtonian fluid ball one obtains for the angular velocity that $\omega<\sqrt{\lambda} / r_{1}$, so it seems reasonable to let $\omega$ go as $\sqrt{\lambda}$. This is also consistent with that $\omega$ or its derivatives only appear quadratically in (9) and (10).

We first consider the post-Minkowskian expansion of the spherically symmetric system. The functions $A$ and $B$ are expanded as

$$
A=1+A_{1}+A_{2}+\ldots, \quad B=1+B_{1}+B_{2}+\ldots,
$$

where subscripts refer to the order in $\lambda$. The freedom of rescaling the time coordinate was used to put the zeroth order term in $A$ to one. We do a similar expansion of the pressure $p=p_{1}+p_{2}+\ldots$ and density $\rho=\rho_{1}+\rho_{2}+\ldots$. Using the field equation (5) we first obtain that $p_{1}$ is a constant. We put this constant to zero so that a zero pressure surface can be obtained. Then

$$
B_{1}=-r \frac{d A_{1}}{d r} .
$$

The first nonvanishing terms of the density and pressure are

$$
\begin{aligned}
& \rho_{1}=\frac{2}{r^{2}} \frac{d}{d r}\left(r^{2} \frac{d A_{1}}{d r}\right) \\
& p_{2}=p_{c}-\left(\frac{d A_{1}}{d r}\right)^{2}-4 \int \frac{1}{r}\left(\frac{d A_{1}}{d r}\right)^{2} d r .
\end{aligned}
$$

To complete the system we have to impose an equation of state $p=p(\rho)$, giving an equation for $A_{1}$. The differentiated version of this equation reads as

$$
\frac{d p\left(\rho_{1}\right)}{d \rho_{1}} \frac{d \rho_{1}}{d r}=-\rho_{1} \frac{d A_{1}}{d r},
$$

where $\rho_{1}$ is given by (72). We note that the total mass $M$ of the configuration will be of the order $\lambda$, while the radius $r_{1}$ will be of zeroth order.

The function $\omega$, which is first order in the rotational parameter $\Omega$, has the post-Minkowskian expansion

$$
\omega=\omega_{1 / 2}+\omega_{3 / 2}+\ldots,
$$

where the indices correspond to the appropriate fractional orders of the quantities in $\lambda$. From the field equation (7) we obtain that $\omega_{1 / 2}$ is a constant. This corresponds to a rigid rotation of the system. It is necessary to keep $\omega_{1 / 2}$ nonzero, since this will provide the nonlinear source term in the higher order equations.

The quantities second order in the rotational parameter, i.e. $h_{2}, m_{2}$ and $k_{2}$, are expanded as

$$
\begin{aligned}
h_{2} & =h_{21}+h_{22}+\ldots \\
m_{2} & =m_{21}+m_{22}+\ldots \\
k_{2} & =k_{21}+k_{22}+\ldots
\end{aligned}
$$

where the second index at each new quantity indicate the order in the post-Minkowskian parameter $\lambda$. To first order in $\lambda$ equations (8), (91) and (10) yield $m_{21}=k_{21}=$ $-h_{21}$ and

$$
\frac{d^{2} h_{21}}{d r^{2}}+\frac{2}{r} \frac{d h_{21}}{d r}-\frac{1}{2}\left(\frac{d \rho_{1}}{d A_{1}}+\frac{12}{r^{2}}\right) h_{21}=\frac{\omega_{1 / 2}^{2} r^{2}}{6} \frac{d \rho_{1}}{d A_{1}},
$$

where

$$
\frac{d \rho_{1}}{d A_{1}}=2 \frac{d^{3} A_{1}}{d r^{3}} / \frac{d A_{1}}{d r}-\frac{4}{r^{2}}+\frac{4}{r} \frac{d^{2} A_{1}}{d r^{2}} / \frac{d A_{1}}{d r} .
$$

We note that a particular solution to eq. (77) is given by $h_{21 p}=-\omega_{1 / 2}^{2} r^{2} / 3$.

Using the condition that $q_{1}$, given by Equation (41), should vanish (to first order in $\lambda$ ) for an asymptotically flat solution and that the corresponding $c_{1}$ is given by $c_{1}=c_{1 p}-c_{1 h} q_{1 p} / q_{1 h}$ as described in Sec. IVD one obtains

$$
c_{1}=-\frac{5 \omega_{1 / 2}^{2}}{\left.48 r_{1}^{5}\left(\frac{d A_{1}}{d r}\right)\right|_{r=r_{1}} ^{5}} \frac{\left.\frac{d}{d r} \log \left(h_{21 h} /\left(r^{4} \frac{d A_{1}}{d r}\right)\right)\right|_{r=r_{1}}}{\left.\frac{d}{d r} \log \left(r h_{21 h} / \frac{d A_{1}}{d r}\right)\right|_{r=r_{1}}} .
$$

Here $h_{21 h}$ is a regular nonzero solution of the homogeneous version of (77). Note that in general $c_{1}$ goes to infinity as $1 / \lambda^{4}$.

From the matching condition (35) it follows that

$$
\left.\frac{d A_{1}}{d r}\right|_{r=r_{1}}=\frac{M}{r_{1}^{2}}
$$

where we assume that the mass $M$ is positive. In 11] it is shown that the rotating fluid ball is oblate in shape if

$$
k_{2 \mid r=r_{1}}+\frac{\xi_{2}}{r_{1}}<0
$$


where $\xi_{2}$ is given by (31). Substitution of $k_{21}=-h_{21}=$ $\omega_{1 / 2}^{2} r^{2} / 3-h_{21 h}$ in (81) together with (80) gives that the fluid ball is oblate iff $h_{21 h \mid r=r_{1}}>0$ to first order in $\lambda$.

For the incompressible case the equations can be integrated completely and give for the asymptotically flat case

$$
\begin{aligned}
& A_{1}=\frac{\rho_{1} r^{2}}{12}+a_{0}, \quad B_{1}=-\frac{\rho_{1} r^{2}}{6}, \quad \omega_{3 / 2}=\frac{\rho_{1} r^{2} \omega_{1 / 2}}{5}, \\
& h_{21}=\frac{r^{2} \omega_{1 / 2}^{2}}{2}, \quad p_{2}=p_{c}-\frac{\rho_{1}^{2} r^{2}}{12} .
\end{aligned}
$$

The integration constant $a_{0}$ can be transformed away by a first order (in $\lambda$ ) rescaling of the time coordinate. The choice $a_{0}=-\rho_{1} r_{1}^{2} / 4$ makes the constant $c_{4}$ in (35) equal to unity. Since $h_{21 h \mid r=r_{1}}=5 \omega_{1 / 2}^{2} r_{1}^{2} / 6>0$ the ball is oblate. From this solution the radius $r_{1}$ of the fluid ball and the parameters $M$ and $a$ of the exterior metric are given by

$$
r_{1}=\frac{2 \sqrt{3 p_{c}}}{\rho_{1}}, \quad M=\frac{4}{3} \frac{\left(3 p_{c}\right)^{3 / 2}}{\rho_{1}^{2}}, \quad a=-\frac{24}{5} \frac{p_{c} \omega_{1 / 2}}{\rho_{1}^{2}}
$$

respectively. The value of $c_{1}$ for asymptotically flat metrics is in this case

$$
c_{1}=\frac{5}{1024}\left(\frac{\rho_{1}}{p_{c}}\right)^{5} \omega_{1 / 2}^{2}
$$

and the relative difference to the Kerr quadrupole moment is

$$
\frac{\Delta Q}{Q}=\frac{25 \rho_{1}}{16 p_{c}}
$$

which diverges as $1 / \lambda$. By scaling the input parameters $\rho_{0 c}, p_{0 c}$ and $\omega_{0}$ in the numerical code used in the previous section as $\lambda, \lambda^{2}$ and $\lambda^{1 / 2}$ respectively, these values are also obtained numerically in the limit $\lambda \rightarrow 0$.

For Newtonian polytropes, $p=p_{c}\left(\frac{\rho}{\rho_{c}}\right)^{\gamma}$, where $\rho_{c}$ and $p_{c}$ are the central density and pressure respectively, the integration of (74) gives the following equation for $A_{1}$

$$
\rho=2 \frac{d^{2} A_{1}}{d r^{2}}+\frac{4}{r} \frac{d A_{1}}{d r}=k\left(a_{1 r_{1}}-A_{1}\right)^{\frac{1}{\gamma-1}}
$$

with $a_{1 r_{1}}=A_{1}\left(r_{1}\right)$ being the value of $A_{1}$ at the zero pressure surface and

$$
k=\left(\frac{\rho_{c}^{\gamma}}{p_{c}} \frac{(\gamma-1)}{\gamma}\right)^{\frac{1}{\gamma-1}} .
$$

The homogeneous version of (77) then becomes

$$
\begin{aligned}
& \frac{d^{2} h_{21 h}}{d r^{2}}+\frac{2}{r} \frac{d h_{21 h}}{d r} \\
& -\frac{1}{2}\left(\frac{-k}{\gamma-1}\left(a_{1 r_{1}}-A_{1}\right)^{\frac{2-\gamma}{\gamma-1}}+\frac{12}{r^{2}}\right) h_{21 h}=0 .
\end{aligned}
$$

From (86) it follows that

$$
\frac{d^{2} A_{1}}{d r^{2}} /\left.\frac{d A_{1}}{d r}\right|_{r=r_{1}}=-2 / r_{1}
$$

on the zero pressure surface $r=r_{1}$. Substitution of this and (80) into (79) now gives

$$
c_{1}=-\left.\frac{5}{48} \frac{r^{5} \omega_{1 / 2}^{2}}{M^{5}} \frac{\left(\frac{d h_{21 h}}{d r} / h_{21 h}-2 / r\right)}{\left(\frac{d h_{21 h}}{d r} / h_{21 h}+3 / r\right)}\right|_{r=r_{1}} .
$$

Let $h_{21 h} \neq 0$ be the solution of (88) that makes the spacetime asymptotically flat. Substitution of $h_{21}=$ $-\omega_{1 / 2}^{2} r^{2} / 3+h_{21 h}$ in (41) then gives

$$
q_{1}=\left.\frac{1}{15 r^{2}}\left(9 h_{21 h}+3 r \frac{d h_{21 h}}{d r}-5 \omega_{1 / 2}^{2} r^{2}\right)\right|_{r=r_{1}}=0
$$

from which the denominator of (90) is

$$
\left.\left(\frac{d h_{21 h}}{d r} / h_{21 h}+\frac{3}{r}\right)\right|_{r=r_{1}}=\frac{5 \omega_{1 / 2}^{2}}{3 h_{21 h}}
$$

Note that $h_{21 h}=0$ would give a non-zero $q_{1}$.

With the identifications $\gamma \equiv 1+1 / n$,

$$
A_{1} \equiv-\lambda \Phi+a_{1 r_{1}}, \quad h_{21 h} \equiv-\frac{\lambda}{2} \phi_{2}
$$

in terms of the functions $\Phi$ (that corresponds to the Newtonian potential for the non-rotating configuration) and $\phi_{2}$ used in [7], and a rescaling of the $r$-coordinate $r \equiv \sqrt{2 / k} s$, with $\bar{k} \equiv k \lambda^{n-1}$, equations (86) and (88) read as

$$
\frac{d^{2} \Phi}{d s^{2}}+\frac{2}{s} \frac{d \Phi}{d s}+\Phi^{n}=0
$$

and

$$
\frac{d^{2} \phi_{2}}{d s^{2}}+\frac{2}{s} \frac{d \phi_{2}}{d s}+\left(n \Phi^{n-1}-\frac{6}{s^{2}}\right) \phi_{2}=0
$$

respectively. Hence these are exactly the same as equations (23) and (41) in the paper [7] by Martín et.al. By rescaling the time coordinate to first order in $\lambda$ the central data for their function $\Phi$ can be met and hence we may rely on their theorem on page 10. From their proof the following inequality holds for regular solutions $\phi_{2}$ of (95)

$$
\frac{\phi_{2}^{\prime}\left(s_{1}\right)}{\phi_{2}\left(s_{1}\right)}-\frac{2}{s_{1}}<0
$$

with $\phi_{2}^{\prime} \equiv d \phi_{2} / d s$ and $s_{1}=\sqrt{k / 2} r_{1}$, or in our notation

$$
\left.\frac{d h_{21 h} / d r}{h_{21 h}}\right|_{r=r_{1}}-\frac{2}{r_{1}}<0 .
$$

That $\phi_{2}\left(s_{1}\right) \neq 0$ and hence also $h_{21 h}\left(r_{1}\right) \neq 0$ is guaranteed by their lemma. But (97) is actually exactly one of 
the factors in the numerator of (90) and hence we conclude that $c_{1} \neq 0$.

Substitution of (92) in (90) now gives that $c_{1}>0$ iff $h_{21 h \mid r=r_{1}}>0$, or equivalently if the fluid ball is oblate in shape. Hence $\Delta Q / Q=16 M^{4} c_{1} / 5 a^{2}>0$ for oblate Newtonian polytropes.

\section{CONCLUSIONS}

It seems that generically the modulus of the quadrupole moment for slowly and rigidly rotating perfect fluid balls is larger than that of the Kerr metric. Also, typically it becomes large for small central pressures. For the incompressible case the ratio $\Delta Q / Q$ is a monotonically decreasing function of central pressure. In the limit, when central pressure goes to infinity, it approaches a small, but positive value. Newtonian polytropes show a slightly more complicated behaviour, and $\Delta Q / Q$ possesses a minimum for $\gamma<2$. However, these minimal values are always larger than 0.2 . The relativistic polytropes behave in a similar way, and the values of the minima are even higher. For linear equations of state something interesting happens. When the constant $d_{1}$ is less than -1 , the configuration becomes infinite in extent for a finite central pressure. In this limit the zero pressure surface approaches the event horizon and $\Delta Q / Q$ goes to zero. Also the central regions of these fluids approach the anti-de Sitter spacetime. All of these solution are quite unphysical since they have a central region with negative density. For the more realistic values of $d_{1}$, i.e. $d_{1}>1$, the curves for $\Delta Q / Q$ show minima, but with values larger than 0.2 .

In a post-Minkowskian approximation the field equations simplify, but still the equation of state can make the problem analytically unsolvable. Generically $\Delta Q / Q$ seems to go to infinity as $\lambda^{-1}$. For the incompressible case the equations can be solved completely. and the result agrees with [6]. We further verify the result of [7] that Newtonian polytropes cannot be matched to Kerr and also show that $\Delta Q / Q>0$ if the rotating configuration is oblate. The differences in approach were essentially that they used global harmonic coordinates and the Lichnerowicz matching conditions, whereas we used the Darmois-Israel matching procedure. The equivalence of the Lichnerowicz and Darmois-Israel matching conditions has been demonstrated in the post-Minkowskian limit for the cases of Wahlquist and polytropes by Cuchí et. al., see [37.

\section{Acknowledgments}

We thank Péter Forgács for helpful discussions. MB gratefully acknowledges the hospitality of the KFKI institute. This work was supported by OTKA-grants No. K61636 and NI68228.
[1] G. Neugebauer and R. Meinel, Astrophys. J. 414, L97 (1993)

[2] J. B. Hartle and K.S. Thorne, Astrophys. J. 153, 807 (1968)

[3] S. Chandrasekhar and J.C. Miller, Mon. Not. R. Astr. Soc. 167,63 (1974)

[4] E. Berti, F. White, A. Maniopoulou and M. Bruni, Mon. Not. R. Astr. Soc. 358, 923 (2005)

[5] W.G. Laarakkers and E. Poisson, Astrophys. J. 512, 282 (1999)

[6] J.A. Cabezas, J. Martín, A. Molina and E. Ruiz, Gen. Rel. Grav. 39, 707 (2007)

[7] J. Martín, A. Molina and E. Ruiz, Class. Quantum Grav 25, 105019 (2008)

[8] W.C. Hernandez, Jr., Phys. Rev. 159, 1070 (1967)

[9] N. Stergioulas, Living Reviews in Relativity, Rotating Stars in Relativity, http://www.livingreviews.org/lrr-2003-3

[10] J. B. Hartle, Astrophys. J. 150, 1005 (1967)

[11] M. Bradley, D. Eriksson, G. Fodor and I. Rácz, Phys. Rev. D., 75, 024013 (2007)

[12] G. Fodor, C.A. Hoenselaers and Z. Perjés, J. Math. Phys. 30, 2252 (1989)

[13] G. Darmois, in Mémorial de Sciences Mathématiques, XXV, Chap. V (1927)

[14] W. Israel, Il Nuovo Cimento BXLIV 4348 (1966)

[15] M.A.H. MacCallum, M. Mars and R. Vera, Phys.Rev. D
75024017 (2007)

[16] G. Fodor, in Relativity today. Proceedings of the Sixth Hungarian Relativity Workshop, 49, Akadémiai Kiadó, ed. Hoenselaers \& Perjés, Budapest (2002) and gr-qc/0612080

[17] M. Bradley, G. Fodor, M. Marklund and Z. Perjés, Class. Quantum Grav., 17, 351 (2000)

[18] M. Bradley, G. Fodor, and Z. Perjés, Class. Quantum Grav. 17, 2635 (2000)

[19] M. Mars and J.M.M. Senovilla, Mod. Phys. Lett. A, 13,1509 (1998)

[20] R. Geroch, J. Math. Phys. 11, 2580 (1970)

[21] R.O. Hansen, J. Math. Phys. 15,46 (1974)

[22] K.S. Thorne, Rev. Mod. Phys 52, 299, (1980)

[23] W. Simon and R. Beig, J. Math. Phys. 24,1163 (1983); F.J. Ernst, in Solutions of Einstein's equations, Techniques and Results, edited by C. Hoenselaers and W. Dietz (springer, Heidelberg, 1984)

[24] T. Bäckdahl and M. Herberthson, Class. Quantum Grav. 22, 3585, (2005)

[25] L.D. Landau and E.M. Lifshitz, The Classical Theory of Fields 4:th ed., Pergamon Press, Oxford (1975)

[26] W. Roos, Gen. Rel. Grav. 7, 431 (1976)

[27] G. Fodor, gr-qc/0011040

[28] H. D. Wahlquist, Phys. Rev. 172, 1291 (1968)

[29] J. M. Whittaker, Proc. Roy. Soc. A 306, 1 (1968)

[30] P. Sarnobat and C.A. Hoenselaers, Class. Quantum Grav. 
23, 5603 (2006)

[31] E. Mukumu, Slowly Rotating Perturbations of the Whittaker Metric, Master's thesis, Umeå University (2008)

[32] H.A. Buchdahl, Phys. Rev. 116, 1027 (1959)

[33] H. Andréasson, J. Diff. Eqs. 245, 2243 (2008)

[34] U.S. Nilsson and C. Uggla Ann. of Physics 286, 292 (2000)

[35] J. M. Heinzle, N. Röhr and C. Uggla Class. Quantum
Grav. 20, 4567 (2003)

[36] A. Lichnerowicz, Théories Relativistes de la Gravitation et de l'Electromagnetisme, Masson, Paris (1955)

[37] J.E. Cuchí, A. Molina and E. Ruiz, Presented at ERE 2008, Salamanca,

http://www.usal.es/ ere2008/website/modules/tinyd0 /content/pdf/Cuchi.pdf 